\title{
An Experimental Study of the Efficiency of Unanimity Rule and Majority Rule
}

\author{
Keith Dougherty* \\ Brian Pitts \\ Justin Moeller \\ Robi Ragan \\ Version 0.4 \\ March 24, 2009
}

\begin{abstract}
We test several claims about the relationship between unanimity rule and Pareto optimality. Buchanan and Tullock (1962), Mueller (2003), and other scholars argue that unanimity rule is more capable of producing Pareto optimal outcomes than other voting rules, such as majority rule, because unanimity rule passes an alternative only if it makes everyone better off. Majority rule can pass alternatives that make some individuals worse off. Dougherty and Edward (2008), in contrast, claim that majority rule is at least as likely to select Pareto optimal outcomes as unanimity rule in finite games if proposals are random, sincere, or strategic. We test the two sets of conjectures in a two dimensional framework using laboratory experiments. Our results suggest: 1) majority rule enters the Pareto set more quickly than unanimity rule, 2) majority rule leaves the Pareto set at the same rate as unanimity rule, and 3) majority rule is more likely to select a Pareto optimal outcome than unanimity rule in the final round of play. Our results also suggest that proposers do not behave observationallyrational in the final round and complete information does not affect the primary result.
\end{abstract}

* All authors: Department of Political Science, University of Georgia, Athens, GA 30602. Corresponding author: (706) 542-2989, <dougherk@uga.edu>. 


\section{Introduction}

Decision making bodies often adjust, and sometimes re-adjust, their voting thresholds between majority rule and unanimity rule ${ }^{1}$. For example, the Treaty establishing a Constitution for Europe recently proposed reducing the unanimity rule requirement in the Council of Ministers to a qualified majority in 26 issue areas. The Financial Accounting Standards Board (FASB) reduced its voting threshold from a supermajority to a majority in 1977 only to increase it back to another supermajority rule in 1991 (King 1994). Each of these changes were an attempt to establish a more desirable threshold. A common criterion for evaluating such voting rules is Pareto optimality. ${ }^{2}$ Most theoretical work concludes that unanimity rule is the best k-majority rule ${ }^{3}$ for promoting Pareto optimality (Buchanan and Tullock 1962; Chen and Ordeshook 1998; Brennan and Buchanan 2000, 151-55; Mueller 2003, 1996). Buchanan and Tullock make it the cornerstone of their seminal work, The Calculus of Consent, and it has been widely accepted ever since. Traditionally viewed, unanimity rule guarantees more efficient outcomes than other voting rules because unanimity rule only passes proposals that

${ }^{1}$ Throughout the paper, we use the term "majority rule" to refer to simple majority rule (i.e. a proposal passes if and only if the yeas exceed the nays) and "unanimity rule" to refer to simple unanimity rule (i.e. a proposal passes if and only if the yeas $>0$ and the nays $=0$ ). See Dougherty and Edward (2004) and Riker (1982) for more details.

${ }^{2}$ Pareto optimality, also known as Pareto efficiency, is the most widely accepted criteria for evaluating efficiency. An alternative is Pareto optimal if no alternative is Pareto preferred to it. That is, no other alternative can make at least one individual better off without making another individual worse off.

${ }^{3}$ A k-majority rule requires at least $k$ individuals to vote in favor of a proposal in order for the proposal to pass, where $N / 2<k \leq N$ and $N$ is the number of individuals; otherwise the status quo is chosen. Two special cases of k-majority rule are majority rule $(k=N / 2)$ and unanimity rule $(k=N)$. See Dougherty and Edward (2004) for more precise definitions when non-voters and "votes to abstain" are permitted. 
make everyone better off. Other k-majority rules, such as majority rule, can pass proposals that make some individuals worse off.

Dougherty and Edward $(2005,2008)$ challenge this claim. They analyze a series of two dimensional spatial voting models where the so-called "chaos" theorem ${ }^{4}$ applies (McKelvey 1976; Schofield 1978). They conclude that majority rule is usually more likely to select a Pareto-optimal outcome than unanimity rule if proposals are random. They also show that majority rule is at least as likely to select Pareto optimal outcomes if proposals are sincere or strategic.

We test these competing sets of theories using laboratory experiments on groups voting in a two dimensional issue space. In particular, we test three primary hypotheses: 1) groups using majority rule are more likely to enter the Pareto set ${ }^{5}$ in fewer rounds than groups using unanimity rule; 2) groups using majority rule are more likely to leave the Pareto set than groups using unanimity rule; and 3) groups using majority rule are at least as likely to select Pareto optimal outcomes at the end of the game as groups using unanimity rule. We also determine whether complete information ${ }^{6}$ affects the relative performance of the two voting rules, and we investigate whether subjects propose rationally in the final round. The final round provides a

${ }^{4}$ The "chaos theorem" states that in n-dimensional space unless ideal points are radially symmetric, no alternative is in equilibrium using pairwise majority rule. In other words, for every alternative in the space, there is another alternative that a majority of individuals prefer to it. Ideal points are radially symmetric around an alternative $\mathrm{x}$ if there is an ordinal, pairwise symmetry of all the ideal points along any vector passing through $\mathrm{x}-\mathrm{a}$ rare condition (Hinich \& Munger 1997, 65).

${ }^{5}$ The Pareto set is the set of all Pareto optimal alternatives.

${ }^{6}$ Complete information refers to situations in which voters know the preferences (in this case ideal points) of the other members of the group. 
particularly clean period for testing such behavior because rational subjects should always make a proposal that moves the outcome closer to their ideal point in the final round (if such an alternative is available).

We find that groups using majority rule enter the Pareto set in fewer rounds than groups using unanimity rule and that groups using majority rule are more likely to select Pareto optimal outcomes than groups using unanimity rule. Such results provide evidence in favor of the theoretical claims of Dougherty and Edward (2008). Contrary to both sets of theories, we find no evidence that groups using majority rule are more likely to leave the Pareto set than groups using unanimity rule. Furthermore, our information conditions do not significantly affect the results, and there is little evidence that subjects propose rationally in the final round. All these results suggest that the widely held relationship between unanimity rule and Pareto optimality needs to be treated much more carefully. Majority rule may be more capable of producing Pareto optimal outcomes in committees, conventions, and legislatures than is traditionally assumed.

\section{Experimental Literature}

There has been a plethora of experimental research on group decision making using majority-rule (Fiorina and Plott 1978; McKelvey and Ordeshook 1981, 1984a, 1984b; Wilson 1986; Bianco et al. 2006, 2008). Some of the earliest works find that groups voting under majority rule are much more predictable than McKelvey and Schofield's chaos theorem might imply. For example, in a two dimensional study McKelvey and 
Ordeshook (1984a) find that if members of a committee are allowed to vote on one dimension at a time, then groups using majority rule are very likely to select the outcome at the median of each separate dimension. This might explain stability in some applied settings, but it avoids multidimensional voting and the brunt of the chaos theorem. Fiorina and Plott (1978), as well as others, examine the more general case where alternatives are chosen over multiple dimensions simultaneously. They find that majority rule equilibria are good predictors of committee behavior when such equilibria exist, and that majority rule outcomes tend toward the center of the ideal points when such equilibria do not exist. ${ }^{7}$

Recent work by Bianco et al. $(2006,2008)$ suggests that the uncovered set ${ }^{8}$ might explain the centralizing behavior of majority rule, particularly in multidimensional cases where no equilibrium exists. Bianco et al. (2006) survey eight experiments on majority rule and find that roughly $94 \%$ of the experimental outcomes fall within the uncovered set. Conducting similar experiments of their own Bianco et al. (2008), find that the percentage of outcomes in the uncovered set range between $59 \%$ and $100 \%$-depending upon the configuration of ideal points. Since the uncovered set is always a subset of the Pareto set (Miller 1980, 80), their evidence supports Dougherty and Edward's (2008) claim that majority rule will select Pareto optimal outcomes

${ }^{7}$ For experiments suggesting that distributive fairness may affect majority rule voting behavior see Miller and Oppenheimer (1982).

${ }^{8}$ Loosely, an uncovered alternative is any alternative that can defeat any other alternative, using majority rule, in two or fewer steps (Penn 2006). 
consistently. ${ }^{9}$ Nevertheless, it does not make the important comparison to unanimity rule that allows us to determine which voting rule is more likely to select a Pareto optimal outcome.

Fewer experiments have been conducted on unanimity rule. Two exceptions include Laing and Slotznick (1991) and Walker et al. (2000). Laing and Slotznick describe a series of single round, unanimity rule experiments in which the core performs better than the Condorect Alternative or the Nash-Harsanyi arbitration outcome.$^{10}$ However, they note that several of their results deviate sharply from the core when the initial status quo is in not in equilibrium.

Walker et al. conduct experiments on two-stage games where experimental groups first select a voting rule (majority rule or unanimity rule), then vote on the allocation of a resource using the method selected. Control groups are assigned majority rule or unanimity rule by the experimenter then vote on the allocation of the resources using the assigned method in the second stage. They find that the experimental groups which are allowed to choose a voting rule select more efficient allocations than control groups that are assigned a voting rule. Furthermore, among

${ }^{9}$ Note that Fiorina and Plott (1978), Bianco et al. (2008), and other authors found cases where majority rule groups did not select Pareto optimal outcomes at the end of their games.

${ }^{10}$ The core is the set of alternatives that cannot be defeated using the specified voting rule. More specifically, in a simple unanimity rule game, an alternative $x$ is a member of the core if there does not exist another alternative $y$ that everyone prefers at least as much as $x$. Under unanimity rule, the Condorcet Alternative (if it exists) is the alternative that is unanimously preferred to all other alternatives. If $q$ is the status quo, the Nash-Harsanyi arbitration outcome is the alternative $z$ that maximizes the product $\prod_{i \in N}\left[u_{i}(z)-u_{i}(q)\right]$. For Euclidean preferences, such an alternative is in the "middle" of the ideal points (Laing and Slotznick 1991). 
experimental groups, the two voting rules produce small differences in the efficiency of group decisions. These findings foreshadow some of the current results.

\section{Theory}

To demonstrate the theoretical differences between traditional claims (Buchanan and Tullock 1962; Mueller 2003; Brennan and Buchanan 2000) and those made by Dougherty and Edward (2008), consider a two dimensional spatial voting framework. Each individual has an ideal point, $l_{i}$, with Euclidean preferences over the alternatives (or policies) within that space. Euclidian preferences imply that each individual prefers an alternative closer to their ideal point more than an alternative further away. Voting proceeds using a forward agenda. The initial status quo $q_{1}$ is paired against a proposal $p_{1}$ in round 1 . The winning alternative in round 1 is then paired against a new proposal

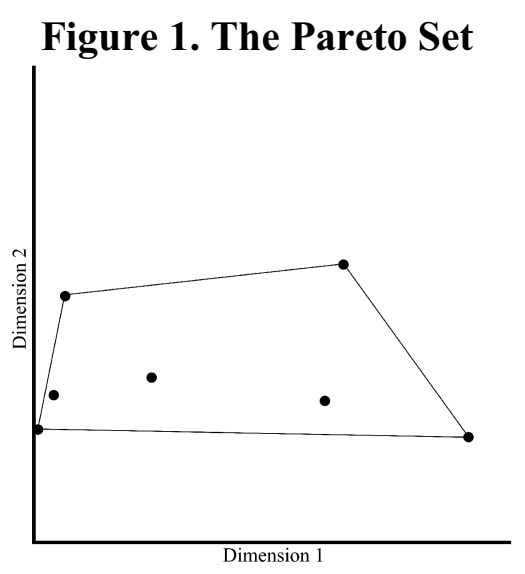

$p_{2}$ in round $2, p_{r}$ in round $r$, and so on, for a total of $\mathrm{R}$ rounds. In what follows, we will refer to both the proposal and its corresponding point in the two-dimensional space as $\mathrm{p}_{\mathrm{r}}$. Similarly, $\mathrm{q}_{\mathrm{r}}$ refers to the status quo in round $\mathrm{r}$. Forward agendas are consistent with 
Tullock's (1998, 70-74) and Mueller's (2003) descriptions of how unanimity rule produces Pareto optimal results. A fixed number of rounds might applies to assemblies with a limited number of amendments and may apply to assemblies which limit the time for make decisions.

In two dimensional space with Euclidean preferences, the Pareto set is the set of alternatives within the convex hull, including alternatives on the edges of the hull. ${ }^{11}$ Figure 1 illustrates these points for a group of seven individuals. ${ }^{12}$ Each dot in the figure represents an individual's ideal point. The polygon connecting the dots outlines the set of Pareto optimal alternatives.

Furthermore, the set of alternatives that cannot be defeated in a single sincere vote is called the core. ${ }^{13}$ For obvious reasons, the core is a widely accepted equilibrium concept.

\subsection{Traditional Claims.}

Under unanimity rule, all points in the Pareto set are in the unanimity rule core, and all points in the unanimity rule core must be in the Pareto set (Ordeshook 1986; Colomer 2001). Consequently, since no proposal can beat a $q_{r}$ in the Pareto set in a single round of voting, no proposal can beat the same $q_{r}$ in a series of voting. In this sense,

${ }^{11}$ More generally, the convex hull is the minimum convex set that contains all ideal points in the space.

${ }^{12}$ These ideal points are from our experimental group 30.

${ }^{13}$ More specifically, alternative $x$ is a member of the unanimity rule core (resp. majority rule core) if there does not exist another alternative $y$ that a unanimity (resp. majority) of individuals prefer to $x$. 
there is a one-to-one correspondence between the unanimity rule equilibria and the set of Pareto optimal alternatives. Hence, it is easy to understand why many scholars believe that in the absence of transaction costs, unanimity rule is particularly adept at producing Pareto optimal results.

Now compare these predictions to majority rule. Using spatial analysis, researchers have demonstrated that unless the distribution of ideal points satisfies radial symmetry (Plott 1967), no alternative in the space is in equilibrium using pairwise majority rule (McKelvey 1976; Schofield 1978). In other words, unless there is radial symmetry, the majority rule core will be empty. If no alternative is in such equilibrium, then for every alternative there is another alternative that a majority prefer to it. Hence, in the absences of an alternative explanation, there would be no clear prediction for majority rule. The only reason that majority rule might end up in the Pareto set would be pure chance.

As Mueller posits, "Once one of these proposals has achieved a unanimous majority no other proposal from the Pareto-efficient set can achieve unanimity when placed against it. Any other proposal must make one voter worse off" (Mueller 2003, p. 139). In contrast, under majority rule, "A coalition of the committee's members could benefit ... by modifying the issues to increase their benefits at the expense of noncoaltion members" (Ibid., p. 140). In other words, majority rule can redistribute resources from one individual to another and change policies from within the Pareto set to policies outside the set. This point is illustrated in Figure 2a. Here seven individuals chose between the status quo $q$ (in the Pareto set) and the proposal $p$ (outside the 
Pareto set). As indicated by the cut line,${ }^{14}$ if the group voted using unanimity rule, it would select the Pareto optimal alternative $q$. If the group voted under majority rule, it would select the Pareto sub-optimal alternative $p$. Since many scholars assume that alternatives are in equilibrium when such an equilibrium exists, it is easy to see why they claim that unanimity rule is better at producing Pareto optimal outcomes than majority rule.

\subsection{Dougherty and Edward}

Dougherty and Edward (2008) do not question these results, but they do arrive at a different conclusion for cases with finite rounds of voting. They base their argument on two key observations: 1) policy outcomes may not be in equilibrium, and 2) the proposal process affects the probability of attaining a Pareto optimal outcome and therefore should be modeled. They consider three types of proposal processes in their study (random, sincere, and strategic), which we briefly summarize.

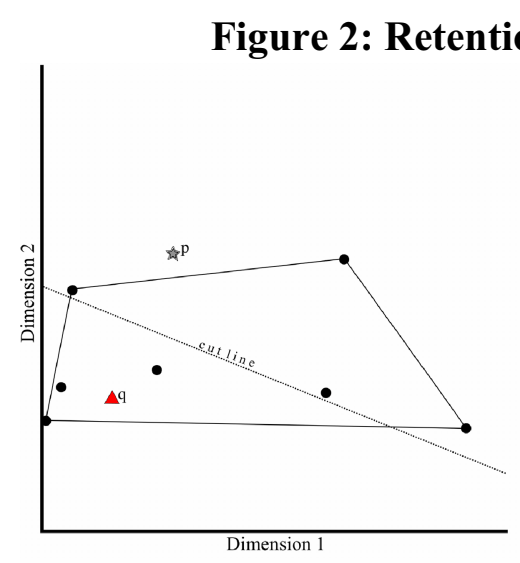

(A)

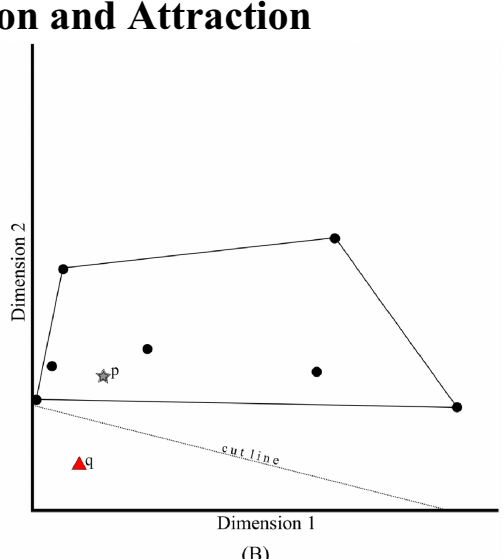

(B)

${ }^{14}$ A cut line demarcates the space between individuals who prefer the status quo and individuals who prefer the proposal. With Euclidean preferences, the cut line is perpendicular to a line between $p$ and $q$ and it intersects the line between $p$ and $q$ at its midpoint. 
First, using simulations, Dougherty and Edward demonstrate that if proposals are random and voting is sincere, then majority rule is more likely to select a Pareto optimal outcome than unanimity rule for several finite series of votes. This is because majority rule requires a lower threshold to move policy toward the set of ideal points and into the Pareto set than unanimity rule (consider Figure $2 b$ ). Of course, unanimity rule is better at keeping policies within the Pareto set. When the forces are combined over the course of $R=1$ to $R=1,000$ rounds, Dougherty and Edward find that majority rule benefits more from its attractive property than unanimity rule does from its retentive property.

Majority rule also has its own weak form of retention. Once the status quo enters the Pareto set, it will rarely leave. For example, imagine a case in which ideal points are uniformly distributed. The status quo is drawn at random from the alternatives within the Pareto set, and the proposal is drawn at random from the alternatives outside the Pareto set. Since the Pareto set is typically located in the center of the space, the proposal outside the Pareto set is typically further away from a majority of voters than is the alternative within the Pareto set. As a result, majority rule leaves the Pareto set far less frequently than one might expect, consistent with the experimental results of Fiorina and Plott (1978), Bianco et al. $(2006,2008)$, and others. Since proposals are random and voting is sincere, this result applies to cases with complete or incomplete information.

Second, Dougherty and Edward analyze sincere proposers using a simple deductive argument. If individuals propose sincerely and vote sincerely, then they will always propose their ideal point, which is by definition within the Pareto set. Therefore, 
no matter which of the two voting rules is used, no proposal will ever be made that is outside of the Pareto set. The only cases that distinguish the performance of the two voting rules are the cases where $q_{1}$ is Pareto sub-optimal. In these cases, the results would be similar to those under random proposing, and majority rule would be at least as likely to move policies into the Pareto set as unanimity rule. As with random proposals, this result applies with both complete and incomplete information.

Third, Dougherty and Edward prove that if information is complete, individuals propose and vote strategically (or propose strategically and vote sincerely), and if the number of rounds is finite, then any k-majority rule will produce a subgame perfect equilibrium that is Pareto optimal. ${ }^{15}$ Majority rule and unanimity rule are simply special cases of this general result. The intuition behind this theorem is that in the final round of voting, rational proposers will propose the alternative closest to their ideal point that will defeat the current status quo. That is, they will propose the alternative closest to their ideal point that is also in the win set of $q_{R^{\cdot}}{ }^{16}$ If the win set is empty or the proposer's ideal point is closest to $q_{R}$, then the proposer will propose a "throw away" alternative making $q_{R}$ the outcome. In the latter cases, $q_{R}$ is Pareto optimal. Since their argument applies to any status quo in the final round of voting (whether Pareto optimal or Pareto sub-optimal), earlier rounds of voting are irrelevant to the proof.

${ }^{15}$ Technically, Dougherty and Edward prove this theorem for alternatives that are at an infinitesimally small distance from the Pareto set. These differences are so imperceptible that they have no bearing on the experiment.

${ }^{16}$ The win set if the set of alternatives that can beat the status quo given the established voting procedure. 


\subsection{Experimental Hypotheses}

The differences between the competing sets of claims suggest five testable hypotheses that we evaluate in our experiment:

H1: Groups using majority rule require fewer rounds to enter the Pareto set than groups using unanimity rule.

This hypothesis is a straightforward implication of Dougherty and Edward's random proposal hypothesis, though it is easy to see how it can apply to sincere or strategic proposers as well. Since the win set of any status quo is larger under majority rule, they presume groups using majority rule will have an easier time moving policies into the Pareto set than those using unanimity rule.

H2: Groups using majority rule will be at least as likely to leave the Pareto set as groups using unanimity rule.

Traditional spatial theory suggests that if a group of rational individuals use unanimity rule, then they will never leave the Pareto set once they enter it. It also suggests that for every $q_{r}$, there is an alternative $x$ that can beat $q_{r}$ under majority rule. As a result, we should expect majority rule groups to be at least as likely to leave the Pareto set as unanimity rule groups - consistent with both sets of theories.

H3: Groups using majority rule will be at least as likely to select a Pareto optimal outcome at the end of the game as groups using unanimity rule.

This is our primary hypothesis, and it is the one which most strongly tests Dougherty and Edward's (2008) claims against earlier observations. Evidence in support of the 
hypothesis would provide evidence in favor of their argument. Evidence against the hypothesis would provide evidence in favor of more traditional claims.

H4: Groups operating under complete information are more likely to select a Pareto optimal outcome at the end of the game than those operating under incomplete information.

This claim is not made by Dougherty and Edward nor authors on the other side of the debate. We include it to help examine behavior that is often difficult to model. It seems that subjects would be more likely to attain a mutually desirable outcome if they know the ideal points of the other members in their group. Complete information might help subjects formulate proposals that will pass, including Pareto optimal proposals. It might also help subjects understand the consequences of their actions and facilitate strategic voting.

For our final hypothesis, we assume: (1) information is complete, (2) $q_{R}$ has a non-empty win set, and (3) $q_{R}$ is not at the same location as the proposer's ideal point. We also define a proposal as observationally rational if and only if the proposer proposes the alternative that is closest to his/her ideal point that is in the win set of $q_{R}$ or is $q_{R}$ if $q_{R}$ is closest. The first three conditions remove cases that do not produce clear predictions about the proposer's behavior. This leads to $H 5$.

H5: If conditions 1-3 are met, subjects will propose observationally rational alternatives in the final round of voting.

It is more difficult to determine whether subjects propose rationally in earlier rounds, because they may have unknown expectations about the behavior of others in 
subsequent rounds. With incomplete information, proposers would be uncertain about the location of the win set and less likely to propose an observationally-rational proposal as we have defined it. If individuals propose rationally, $\mathrm{H} 5$ should apply to both majority rule and unanimity rule groups.

\section{Experimental Design}

Subjects were recruited from various sections of Introduction to American Government courses at a large public university and participated during regular class time.

Participating classes were typically divided into four groups of seven subjects each and assigned a voting rule (either majority rule or unanimity rule) and an information condition (complete or incomplete). Hence, a typical class contained the following groups: majority, complete; majority, incomplete; unanimity, complete; and unanimity, incomplete. Complete information means subjects were given a two dimensional graph that contained the ideal points of the other subjects as well as their own ideal points and the location of the status quo. The subject's own ideal point was presented in a different color and shape to avoid confusing it with the other points. Subjects given incomplete information were given a similar two dimensional graph that contained only their own ideal point and the initial status quo. Each set of four groups (majority, complete; majority incomplete; unanimity, complete; and unanimity, incomplete) were assigned the same set of ideal points so that ideal points matched across treatment conditions. This practice was maintained even if there were not enough students in a class to run four groups on the same day. The experiment included 32 total groups 
with eight different sets of ideal points (see Appendix C). Each set of ideal points were randomly drawn from a uniform distribution on a $100 \times 100$ square until the initial status quo, always $(10,10)$, was Pareto sub-optimal. For the last third of our experimental groups, we also excluded draws which contained $(50,50)$ in the convex hull. The latter helped prevent a natural focal point from affecting the results.

At the start of each class, the principal investigator read general instructions which described the purpose of the experiment and what subjects would do if they participated (see Appendix A). Subjects were also told about the payment schedule and the minimum and maximum payment they would receive based on the distance between the final outcome and their ideal point. They were further informed that there was no chance of losing money and that each subject would receive a minimum of $\$ 1$. After the brief introduction, subjects were given consent forms. Subjects agreeing to participate signed the form, were randomly assigned to one of the four groups, and received a manila envelope containing instructions for the experimental rounds, a brief questionnaire, and a payment form. Attached to the back of the envelope were instructions for the practice rounds and a piece of string (to measure distance to ideal points). Students declining to participate were asked to stay and observe the proceedings for educational purposes or to volunteer as a time keeper or a data recorder. Participating subjects then followed the experimenter assigned to their group to a separate room where the experiment was conducted. Each group of seven participated in a different room.

After relocating, subjects were asked to read the directions on the back of the manila envelope for the practice rounds (these directions were similar to those in 
Appendix B). Subjects were reminded there should be no talking during the experiment and were given five minutes to read the instructions and identify their ideal point for the practice rounds. After the subjects read the instructions, the group leader re-described proposing, voting, payment, and other experimental procedures (see Appendix A). Subjects were then given another opportunity to ask questions.

The practice rounds were identical to the experimental rounds except that experimental rounds lasted for 10 rounds (while the practice rounds lasted for 4 rounds), the experimental rounds started with a different status quo $((10,10)$ instead of $(80,80)$ for the practice rounds), and the experimental rounds utilized different ideal points. To start the experiment, the group leader stated the initial status quo and asked if anyone wanted to make a proposal. Subjects could propose any $(x, y)$ pair between 0 and 100 inclusive, in increments of .01. A subject could propose in future rounds, but only after everyone in the group was given the opportunity to propose first, if they desired. Subjects were given 30 seconds to consider the two alternatives (the proposal and the status quo). They then voted by a show of hands that all subjects could see. If the proposal passed, the proposal would become the new status quo for the next round of voting. As proposing and voting proceeded, the group leader recorded the subject's identification number, their proposal, and the status quo on a chalk board in the front of the room.

At the end of the fourth practice round, students were instructed to return the materials for the practice rounds to the group leader. Subjects were then asked to open their sealed envelope and read over the three page instruction instructions detailing the experimental rounds (see Appendix B). The questionnaire and payment 
form remained in the packet. Subjects were allowed three to five minutes to read through these instructions and locate their new ideal point. The third page of the instructions contained the subject's new ideal point and depending on the information condition, the ideal points of the other participants. After the subjects examined the materials, the group leader reemphasized that payment was based on the distance between the subject's ideal point and the final outcome after ten rounds of voting. The experimental rounds were then conducted following the same procedure as the practice rounds.

When the experimental rounds had ended, subjects were asked to complete the two remaining sheets in the envelope (a payment form and a subject questionnaire). The payment form asked information needed to pay the subjects by check. The subject questionnaire asked for general biographic information such as gender, ethnicity, academic major, and year of college (see Appendix B). When subjects completed these forms they returned all materials to the manila envelope and gave it to their group leader. Subjects were then given the option of returning to the main classroom where they could learn the exact amount of their earnings, or to leave and learn their payment when they received it in the mail.

\section{Results}

The results of the experiment are largely consistent with the claims of Dougherty and Edward (2008). See Appendix C. ${ }^{17}$

\footnotetext{
${ }^{17}$ A total of 224 subjects participated in the study: $64 \%$ were freshman, $24 \%$ sophomores, $8 \%$ juniors, and $4 \%$ seniors. Since American Government is required of all
} 
H1: Rounds to Enter the Pareto Set. Of the 32 experimental groups, 24 of them entered the Pareto set for at least one round. Of those 24 groups, those using majority rule entered the Pareto set in an average of 1.7 rounds. Groups using unanimity rule entered the Pareto set considerably more slowly in an average of 5 rounds. This difference is statistically significant at the .01 level. Such data provide strong evidence in favor of Hypothesis 1.

H2: Leaving the Pareto Set. We also found evidence in favor of Hypothesis 2. As traditional equilibrium models would predict, none of the groups that used unanimity rule left the Pareto set after they entered it. More surprisingly, however, none of the groups using majority rule left the Pareto set after they entered it, contrary to the predictions of both groups of theorists.

One might conjecture that our subjects did not have ample opportunity to leave the Pareto set because Pareto sub-optimal alternatives were rarely within the win set of the status quo. However, this is not the case. We can evaluate the conjecture by looking at the number of cases where subjects vote under majority rule and the win set extends beyond the Pareto set. Of the 135 rounds where a status quo was in the Pareto set, the win set extended beyond the Pareto set in 106 cases (79\%). In these 135 cases, the proposer proposed an alternative outside the Pareto set only 18 times $(13 \%){ }^{18}$ In other words, there were many sub-optimal alternatives that could beat a

students at the university, majors varied greatly. The modal major was in the category of health, medicine, or veterinary sciences (42 subjects), while only 17 majored in a social science. Women were $70 \%$ of the subjects.

${ }^{18}$ Of the 18 cases where the status quo was Pareto optimal and the win set extended beyond the Pareto set, only one proposer proposed an alternative in the win set $(6 \%)$. This 
Pareto optimal status quo under majority rule, but these alternatives were rarely proposed.

Looking only at the final rounds (where for majority rule groups the status quo was always in the Pareto set), the win set extended beyond the Pareto set in 11 of the 16 majority rule cases $(69 \%)$. In these 11 cases, the proposer proposed an alternative outside the Pareto set only twice. In one of the cases with a sub-optimal proposal (group 21), the proposal was outside the win set and defeated (2-5). In the other, a sub-optimal proposal was made within the win set, but it was so close to the edge of the win set that it was also defeated (3-4).

Three mechanisms help to explain this phenomenon. First, if subjects proposed sincerely, then they would always propose their ideal point and this would often fail. In cases where it passed, the proposal would be Pareto optimal. Second, if subjects proposed randomly, then they could propose Pareto sub-optimal alternatives that could pass, but that does not mean that such proposals would be likely. With only ten rounds of voting, random proposers may have simply been "lucky" enough to have never proposed a winning alternative outside the Pareto set. Third, if subjects proposed strategically as if each round were the last round and they also assumed that other subjects would vote strategically (or sincerely) as if it was the last round, then it would be rational for the proposer to propose an alternative within the Pareto set (as shown by Dougherty and Edward's Theorem 2, 2008). All three explanations might explain why

proposal passed but the group ultimately returned to the Pareto set before the game had ended. 
proposers in majority rule groups almost never proposed an alternative that would move their group outside of the Pareto set even when there are ample opportunities.

H3: Majority Rule is at Least as Likely to Select Optimal Outcomes. We found strong support for our primary hypothesis, which corroborates Dougherty and Edward's (2008) claims. As mentioned previously, all of the majority rule groups ended the experiment in the Pareto set. Only half of the unanimity rule groups ended the experiment in the same set. The difference between the groups is statistically significant at the .01 level, indicating that majority rule is more likely to select a Pareto optimal outcome than unanimity rule. Dougherty and Edward may have found an explanation for this in their simulations. Unanimity rule groups frequently stay at the initial status quo or a status quo they arrive at in a subsequent round because it is very difficult to find alternatives that pass unanimously. Among the 16 unanimity rule groups, six remained at the initial status quo (which was sub-optimal) while two more moved to a different sub-optimal point and remained at that point for the remainder of the game. As a result, half of the unanimity rule groups never entered the Pareto set, compared to the majority rule groups which all entered the Pareto set and ironically stayed.

This provides evidence against the long standing notion that unanimity rule is particularly adept at producing Pareto optimal outcomes. Even though majority rule is not in equilibrium in the Pareto set, subjects in our majority rule groups never left the set once they entered it. Although this result is surprising, it is somewhat consistent with experiments on the uncovered set (Bianco et al. 2006, 2008). Furthermore, even 
though unanimity rule is in equilibrium within the Pareto set, our results suggest that subjects using unanimity rule may not attain such an equilibrium.

H4: Information and the Pareto Set. We do not find evidence in support of Hypothesis 4. Of the 16 complete information groups, 13 ended the experiment in the Pareto set $(81 \%)$. In contrast, 11 of the 16 incomplete information groups ended the experiment in the Pareto set $(69 \%)$. This difference is not statistically significant, with a one tailed $p$-value of 0.22 . To determine whether the type of rule masked the effect of our information conditions, we also ran the following least squares regression:

$$
\text { pareto }=\beta_{0}+\beta_{1} \text { majority }+\beta_{2} \text { complete }+\epsilon
$$

where pareto $=1$ if the group outcome was in the Pareto optimal set in the final round ( 0 otherwise); majority = 1 if the group voted using majority rule (= 0 if unanimity); and complete $=1$ if the group was given complete information ( $=0$ if incomplete $) \cdot{ }^{19}$ Majority rule had a positive and statistically significant effect at the .01 level, consistent with our findings for H1. However, even when the type of voting rule is controlled, the information condition did not have a statistically significant effect on whether a group selects a Pareto optimal outcome in the final round. This again suggests that the information condition did not have a significant effect on the results. Groups often selected Pareto optimal outcomes even if the ideal points of the other subjects were unknown.

${ }^{19}$ Regression estimates were as follows with standard errors in parentheses underneath: pareto $=0.438+0.500$ majority +0.125 complete
(0.112) (0.129)

Neither logit nor probit regression can be used because majority $=1$ predicts pareto $=1$ perfectly, thus preventing the maximum likelihood estimate from converging. 
H5: Observationally-Rational Proposing. We also fail to find evidence in support of Hypothesis 5. Of our 16 complete information groups, 11 had status quos in the final round that 1) contained a non-empty win set and 2) were not at the same location as the proposer's ideal point. ${ }^{20}$ Among these, none of the final round proposers proposed an alternative that would be deemed observationally-rational. Few even came close. If we define close as within 5 units of the observationally-rational proposal, then only two final round proposals would be deemed close. If individuals were universally rational, we would expect all 11 of the final round proposers in complete information groups with non-empty win sets and status quos not at their ideal points to propose observationallyrationally or at least within that vicinity. This provides fairly strong evidence against $H 5$. It also raises questions about the rationality of proposers in earlier rounds where rational proposing is more difficult to determine, but equally important for the results.

\section{Conclusion}

Since Buchanan and Tullock's seminal work (1962) The Calculus of Consent, unanimity rule and Pareto optimality have been seen as almost synonymous. ${ }^{21}$ Their claim has been reinforced by equilibrium analysis in multidimensional spatial voting games (Colomer 2001; McKelvey 1976; Schofield 1978), where it has been shown that unanimity rule equilibria are Pareto optimal and majority rule equilibria rarely exist.

${ }^{20}$ Of course, the remaining 5 were unanimity rule groups that were within the Pareto set in the final round. Hence, their win sets were empty.

${ }^{21}$ For example, Buchanan $(1967,285)$ writes, "it is evident that [unanimous consent] is the political counterpart of the Pareto criterion for optimality." 
Since Shepsle $(1979,1986)$, the dominant explanation for the stability of outcomes produced by majority rule has been institutional, not something related to the voting rule itself or the incentives of the proposer. Combined, the perception is that unanimity rule produces efficient outcomes and, without institutional constraints, majority rule produces almost anything.

Our experiments suggest the opposite. We demonstrate that unanimity rule may not be particularly adept at selecting Pareto optimal outcomes if the starting point is not in equilibrium and that majority rule does not leave the Pareto set as might be expected. Testing 32 groups of subjects with forward agendas, changing proposers, and finite rounds of voting, we find that all of our majority rule groups were in the Pareto set in the final round of the game and none of these groups left the Pareto optimal set as commonly anticipated. In contrast, our unanimity rule groups were less likely to enter the Pareto set than majority rule because members of unanimity rule groups found it difficult to formulate proposals that everyone preferred to the status quo. In other words, majority rule groups did not "wander anywhere" and unanimity rule groups occasionally got stuck where they began. These results are consistent with Dougherty and Edward's (2008) claim that the proposal process is important and out of equilibrium starting positions can affect theoretical claims.

Of course, every institution and every set of instructions in an experimental design can alter the results. When comparing our experiment to other experiments on majority rule behavior it is important to keep our stopping rule in mind. Fiorina and Plott (1978) and Bianco et al. (2008) stopped their groups when a majority of subjects within the group voted to adjourn. Our groups adjourned after exactly ten rounds. We did this 
to test a conjecture about finite agendas and fully admit that it may have affected the results. Nevertheless, subjects voting to stop a game have to be constantly concerned about the game ending prior to a fixed stopping point, such as our $10^{\text {th }}$ round, and they may have less opportunity to propose and vote strategically with this expectation in mind. Furthermore, voting to adjourn seems to establish expected values that are more akin to finite agendas than to infinite agendas. Hence, different stopping rules might effect the results, but those results should not differ in kind.

Standard game theory makes it clear that unanimity rule is the ideal voting scheme for maintaining Pareto-optimal outcomes (Colomer 2001, pp. 71-73). However, our experiments suggest that majority rule is better at obtaining Pareto-optimal outcomes than unanimity rule. This is because the strong attractiveness property of majority rule makes up for its weaker retentiveness property. Institutional framers who want to obtain Pareto-optimal outcomes will want to consider these differences before they impose unanimity rule as a method of finding optimal quantities of public goods (Cornes and Sanlder 1996; Lindahl [1919] 1967), the best allocation of resources in a legislature (Aldrich 1995; Niou and Ordeshook 1985), or efficient voting rules for an assembly (Hardin 1999; Buchanan and Tullock 1962). 


\section{Appendix A: Verbal Instructions}

\section{General Instructions (before breaking into groups)}

We are going to conduct an experiment on the properties of voting rules in two dimensional spatial voting models. This should help us test some theories about the differences between majority rule and unanimity rule and help you get exposure to some of the science behind political science.

Subjects who chose to participate will be randomly assigned to one of _(4) groups. We will then run out experiments in these groups - one voting under majority rule, another voting under unanimity rule for 10 rounds. When the experiments are done, you will fill out a brief questionnaire and payment form. You will get paid based on the distance between the final outcome and your ideal point as outlined in the instructions. The maximum you can get paid is $\$ 15$. The least you will get paid is $\$ 1$. We will tell you how much you will get paid at the end of the experiment.

We will now pass out two consent forms (one for you and one for us). If you are interested in participating, please read and fill out the consent forms.

We will now go around the room and assign subjects to groups. Please keep your packets face down until we ask you to turn them over. Your participation is entirely voluntary. If you do not want to participate, please say so when we come to you, and we will pick up your forms. We can only use _(28)_ of you today, so unfortunately we will have to skip some of you. We ask that students who do not participate stay in the class and observe the proceedings for educational purposes. You might even be able to help us keep time or record information in the experiment, if you'd like. If you do help us, then we will give you your choice of a candy bar or a breakfast bar as a token of our appreciation.

The leader of your group will describe the method of proposing alternatives and how voting will proceed. We hope you enjoy the experiment.

\section{Majority Rule Instructions (group leader: upon arrival in experimental room)}

Please turn over your packets and read the instructions for the practice rounds. We will be voting for 4 rounds so that you can get the hang of what we will be doing in the experimental rounds. There should be no talking, or other communication, during the experiment [pause: roughly 5 minutes for instruction reading].

Voting will proceed as follows. Any one of you can raise your hand and propose an alternative, which is identified by any pair of numbers between 0 and 100 inclusive (real numbers in increments of .01 are acceptable). For example, you may propose $(50,0)$ or you may propose $(.01, .81)$. Proposal values can be repeated as many times as the group wants but each person will be allowed to propose once before anyone is allowed to propose a second or a third time. To propose, first state your identification number (on the top left hand corner of the payoff page), then the $x$ and $y$ 
values of your proposal (between 0 and 100 inclusive). After a proposal is made we will allow 30 seconds for members of your group to think about the choices. We will then vote on the proposal versus the status quo (the alternative we have now). A proposal passes if more subjects vote yea than nay. If the proposal passes, it will become the new status quo. If it does not pass, then the status quo remains the same. In the practice rounds, voting continues for exactly 4 rounds. In the $4^{\text {th }}$ round, voting stops. If this were the actual experiment, you would be paid based on the distance between the alternative selected at the end of the game and your ideal point. The closer the final alternative is to your ideal point the more you will get paid. The exact amount you will get paid will be presented before the experimental rounds.

Any questions?

There is no talking or communication during the experiment. We also ask that you cover-up your paper so that others don't see your ideal point. [run 4 practice rounds]

Please pass your papers related to the practice rounds to the front of the room. [after collecting] Please open your manila envelops and pull out the stapled papers (this should be the first of three forms in the envelop. The other two are a brief questionnaire and a payment form that you will fill out later). Please take a moment and read over the instructions on the stapled sheet. [pause: roughly 3 minutes].

We will now vote in the experimental rounds following the same procedures as we did before. However, voting will continue for exactly 10 rounds, and you have probably received a new ideal point. Your ideal point is on the third page, not the second page. The second page only helps you to understand the payment schedule. You will get paid based on the distance between the alternative selected at the end of the game (on the third page) and your ideal point. The closer the final alternative is to your ideal point the more you will get paid. If the final outcome is at your ideal point, you will receive the maximum amount which is $\$ 15$. If the final outcome is extremely far away from your ideal point, you will receive the minimum payment of $\$ 1$.

Any questions?

[run experimental rounds -10]. Now that the experiment is over, there are two things that we need you to do before we return to the original room. First, pull out the remaining sheets of paper from the envelop and answer the questions on the brief questionnaire. Your responses are voluntary. [pause momentarily]. Second, please fill out your payment form completely, including your group number and subject id number on the top. If you would like to get paid, we need your social security number for tax purposes. Ten digit student id numbers cannot be used for this purpose. We go to great lengths to protect your social security number. For example, as soon as your payment is processed, this form will be shredded, and no record of your personal information will be kept with experimental information.

[when finished] Please put all your materials back into you manila envelop. If you would like to know how much you will be paid, please follow me back to your 
classroom. If you want to leave now, please hand me your packet. In either case, you will receive a check in the mail shortly. Thanks for participating.

\section{Appendix B: Written Instructions (Experimental Rounds)}

Available Upon Request

\section{Appendix C: Ideal Points and Outcomes}

Available Upon Request

\section{References}

Aldrich, John. 1995. Why Parties?: The Origin and Transformation of Party Politics in America. Chicago: University of Chicago Press.

Bianco, William T., Michael S. Lynch, Gary J. Miller, and Itai Sened. 2006. “'A Theory Waiting to be Discovered and Used': A Reanalysis of Canonical Experiments on Majority Rule Decision Making" Journal of Politics, 68(4): 837-850.

Bianco, William T., Michael S. Lynch, Gary J. Miller, and Itai Sened. 2008. "The Constrained Instability of Majority Rule: Experiments on the Robustness of the Uncovered Set" Political Analysis 16(2):115-137.

Brennan, Geofrey and James M. Buchanan. 2000. The Reason of Rules. Indianapolis, Liberty Fund.

Buchanan, James M. 1967. Public Finance in Democratic Process. Chapel Hill: The University of North Carolina Press.

Buchanan, James M. and Gordon Tullock. 1962. The Calculus of Consent. Ann Arbor: The University of Michigan Press.

Chen, Yan, and Peter C. Ordeshook. 1998. "Veto Games: Spatial Committees under Unanimity Rule." Public Choice 97(4): 617-43. 
Colomer, Josep H. 2001. Political Institutions: Democracy and Social Choice. New York: Oxford University Press.

Cornes, Richard, and Todd Sandler. 1996. The Theory of Externalities, Public Goods, and Club Goods, 2nd ed. New York: Cambridge University Press.

Dougherty, Keith L. and Julian Edward. 2004. "The Pareto Efficiency and Expected Costs of k-Majority Rules." Politics Philosophy and Economics 3(2): 161-89.

Dougherty, Keith L. and Julian Edward. 2008. "Voting for a Pareto Efficient Constitution." (University of Georgia).

Fiorina, Morris P., and Charles R. Plott. 1978. "Committee Decisions under Majority Rule: An Experimental Study." American Political Science Review 72(2): 575-98. Hardin, Russell. 1999. Liberalism, Constitutionalism, and Democracy. New York: Oxford University Press.

Hinich, and Melvin J. and Michael C. Munger. 1997. Analytical Politics. New York: Cambridge University Press.

Laing, James D. and Benjamin Slotznick. 1991. "When anyone can veto: a Laboratory Study of Committees Governed by Unanimous Rule." Behavioral Science 36(3): $179-95$.

Lindahl, Erik. [1919] 1967. "Just Taxation—A Positive Solution," in Classics in the Theory of Public Finance, edited by R. Musgrave and A. Peacock. New York: St. Martin's Press, pp. 168-76.

McKelvey, Richard. 1976. "Intransitivities in mulitdimensional voting models and some implications for agenda control." Journal of Economic Theory 12:472-82. 
McKelvey, Richard D. and Peter C. Ordeshook. 1981. "Experiments on the Core: Some Disconcerting Results for Majority Rule Voting Games" The Journal of Conflict Resolution, 25(4): 709-724.

McKelvey, Richard D. and Peter C. Ordeshook. 1984a. "An Experimental Study of the Effects of Procedural Rules on Committee Behavior." Journal of Politics 46(1): 182-205.

McKelvey, Richard D. and Peter C. Ordeshook. 1984b. "Rational Expectations in Elections: Some Experimental Results Based on a Multidimensional Model." Public Choice 44(1): 182-205.

Miller, Gary J., and Joe A. Oppenheimer. 1982. "Universalism in Experimental Committees." American Political Science Review 76(3): 561-74.

Miller, Nicholas. 1980. "A New Solution Set for Tournament and Majority Voting." American Journal of Political Science 24 (1): 68-96.

Mueller, Dennis. 1996. Constitutional Democracy. New York, Oxford University Press.

Mueller, Dennis. 2003. Public Choice III. New York, Cambridge University Press.

Niou, Emerson and Peter Ordeshook. 1985. "Universalism in Congress." American Journal of Political Science 29(2): 246-58.

Ordeshook, Peter. 1986. Game Theory and Political Theory. New York, Cambridge University Press.

Walker, James M., Roy Gardner, Andrew Herr, Elinor Ostrom. 2000. "Collective Choice in the Commons: Experimental Results on Proposed Allocation Rules and Votes" The Economic Journal 110(460): 212-234. 
Penn, Elizabeth Maggie. 2006. "Alternate Definitions of the Uncovered Set and their Implications" Social Choice Welfare 27: 83-87.

Plott, Charles R. 1967. "A Notion of Equilibrium and its Possibility under Majority Rule." American Economic Review 57:787-806.

Riker, William H. 1982. Liberalism against Populism. Prospect Heights, IL: Waveland Press.

Schofield, Norman J. 1978. "Instability of simple dynamic games." Review of Economic Studies 45:575-94.

Tullock, Gordon. 1998. On Voting: A Public Choice Approach. Northampton, MA, Edward Elgar.

Wilson, Rick K. 1986.“Forward and Backward Agenda Procedures: Committee Experiments on Structurally Induced Equilibrium." Journal of Politics 48(2): 390-409. 